\title{
Bioanalysis
}

\section{Recommendations for clinical biomarker specimen preservation and stability assessments}

With the wide use of biomarkers to enable critical drug-development decisions, there is a growing concern from scientific community on the need for a 'standardized process' for ensuring biomarker specimen stability and hence, a strong desire to share best practices on preserving the integrity of biomarker specimens in clinical trials and the design of studies to evaluate analyte stability. By leveraging representative industry experience, we have attempted to provide an overview of critical aspects of biomarker specimen stability commonly encountered during clinical development, including: planning of clinical sample collection procedures, clinical site training, selection of sample preservation buffers, shipping logistics, fit-for-purpose stability assessments in the analytical laboratory and presentation of case studies covering widely utilized biomarker specimen types.

First draft submitted: 16 January 2017; Accepted for publication: 6 March 2017; Published online: 15 May 2017

Keywords: biomarker preservation $\bullet$ biomarker specimen collection $\bullet$ biomarker stability

In an effort to accelerate drug development, the US FDA launched The Critical Path Initiative [1], whose primary goal was to drive utilization of biomarkers in drug development. Identification and monitoring of biomarker levels in response to therapeutic interventions has become an integral tool for provision of critical information at various stages of drug development. In order to effectively utilize biomarkers to draw meaningful conclusions, it is imperative that the measured concentration of the analyte in study samples is as close as possible to the actual analyte concentration at the time of sample collection. Therefore, optimal preservation of biomarker specimens from collection to testing becomes paramount. However, this seemingly simple goal is not a trivial undertaking. It is given that analytically well qualified, fit-for-purpose analytical methods should be used for quantification of the biomarkers in study samples. However, there are many potential sources of variability in biomarker quantification that are beyond the con- trol of a bioanalytical laboratory [2-4], namely: inter- and intra-individual biological variability due to genetic make-up, dietary intake, circadian rhythm and environmental conditions; type of biological specimen (e.g., tissues, cells, serum, plasma, RNA and DNA); sample collection, processing and storage at the clinical site; shipment and transportation of samples from the clinical site to the bioanalytical facility; handling and storage of these samples in the bioanalytical laboratory (Figure 1).

These endogenous analytes, especially protein biomarkers, are prone to degradation and deactivation under some conditions of sample collection, processing and storage. Therefore, it is of utmost importance that well defined processes and procedures, along with a comprehensive clinical site personnel training program, are strictly implemented. Furthermore, reliable and robust bioanalytical methods should be made available before embarking upon a biomarker evaluation program. Based upon working knowledge of clinical develop-
Naveen Dakappagari*,1, Hui Zhang $^{2}$, Laurie Stephen ${ }^{3}$, Lakshmi Amaravadi $^{4}$

\& Masood U Khan ${ }^{5}$

'Navigate Biopharma Services, Inc.,

A Novartis Company, 1890 Rutherford Rd, Carlsbad, CA, USA

${ }^{2} \mathrm{QPS}, \mathrm{LLC}, 1$ Innovation Way, Newark, $D E$, USA

${ }^{3}$ Ampersand Biosciences LLC, 3 Main St. Saranac Lake, NY, USA

${ }^{4}$ Genzyme, a Sanofi Company,

500 Kendall Street Cambridge, Boston, MA USA

${ }^{5}$ Alliance Pharma, 17 Lee Boulevard,

Malvern, PA, USA

*Author for correspondence:

Tel.: +1 7605166430

Fax: +1 7605166201

naveen.dakappagari@navigatebp.com 


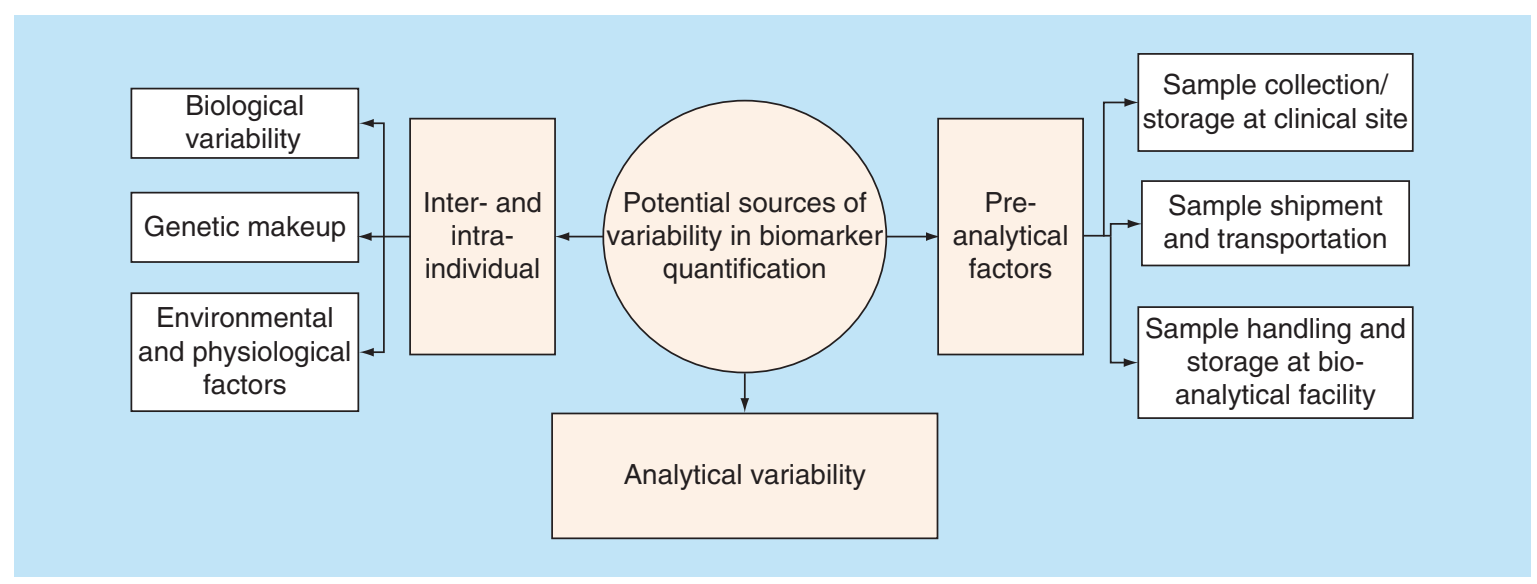

Figure 1. Factors contributing to biomarker instability.

ment programs and collective experience in bioanalytical laboratory settings, the authors have identified potential barriers to optimal preservation of biomarker specimens. We made a first attempt to provide general guidance spanning the entire spectrum of a biomarker evaluation program from preanalytical work flow to clinical site training, as well as laboratory practices for evaluating fit-for-purpose stability of biomarkers in the most widely utilized specimen types, namely, biological fluids, cells and tissue specimens. Readers are encouraged to look at additional references for a deeper discussion of specific case studies.

\section{Preanalytical stability considerations}

The sample collection procedure must be designed to ensure preservation of the analyte. Today's clinical trials are conducted globally, spanning many countries and regions, varying from small clinics to major medical centers. Similarly, investigator sites have varied levels of expertise and infrastructure. Therefore, it is critical to design biomarker sample collection manuals commensurate with the realities on the ground.

\section{Creating a study-focused biomarker sample collection manual}

A successful sample collection procedure is devised with close attention to the study protocol and the biomarker method performance results. The sample collection procedure should provide both a global overview and detailed instructions that guide the clinical staff through all necessary steps, namely, specimen tube labeling, collection procedures, temporary storage, proper packaging and shipping of the samples. If possible, the biomarker assay should have already been thoroughly evaluated at the time of formulating the sample collection procedure, so as to effectively address any stability considerations. However, the practical aspects of sample collection in specific situations may influence the choice of matrix and ultimately assay method development and validation. Sample collection procedures and instructions may be generated by the study sponsor, a project manager from a contract research organization, or trial site personnel. Regardless of the originator, an experienced bioanalyst should be consulted to ensure the samples collected are fit for biomarker analysis. A list of key elements for inclusion in a biomarker sample collection manual is outlined below.

\section{Preparation of sample labels \& manifests}

The overall sampling plan including types of matrices, number of specimen tubes and chronology of collections should trigger generation of sample labels and manifests. These documents must be re-examined and realigned with future amendments of clinical protocols.

\section{Specimen collection \& handling}

Depending on the clinical study logistics, specimen collection materials may be directly obtained by the clinical trial site, provided to the site in bulk or packaged as individualized patient sample collection kits. The assay validation data and the study protocol should dictate the blood collection tube type and size [5]. Adequate sample collection materials (e.g., labels, transfer pipets and sample storage tubes) should be included to facilitate a smooth sample collection process and subsequent storage. Whenever possible, samples should be divided into two aliquots and stored independent of each other to avoid inadvertent total loss. Additionally, if freezethaw instability is observed during assay development, each sample should be split into multiple aliquots to facilitate repeat testing. Provision for multiple aliquot storage should be reflected in the number of sample tubes and labels provided in the collection kits. Specialized specimen collection procedures (e.g., peripheral blood mononuclear separations) may be best conducted at a central laboratory to maximize uniformity 
of sample treatment and minimize the influence of preanalytical variables on biomarker quantification.

\section{Concise instructions}

A set of 'step-by-step' sample collection instructions that describes all the activities in the process, from labeling of tubes to final storage of the specimens, along with diagrammatic aids should be provided. These instructions should reflect the procedures already demonstrated to maintain sample stability during processing as outlined in Table 1.

\section{Long-term storage}

Biological specimens are typically stored frozen at -20 or $-70^{\circ} \mathrm{C}$, according to their documented or anticipated stability. At the initiation of a clinical study, extended sample storage stability is often unavailable. In this situation a conservative approach must be adopted and samples should be stored at the default temperature of $-70^{\circ} \mathrm{C}$ or lower, while continuing the stability program. Moreover, many clinical sites involved in late-stage studies have limited sample storage capability, particularly at $-70^{\circ} \mathrm{C}$. These factors must be accommodated by the sample shipping schedule. Ideally, clinical sites should attempt to organize the biomarker specimen shipments in a manner that minimizes additional sample handling and sorting by the bioanalytical laboratory.

\section{Sample shipment}

Prior to initiation of a biomarker specimen shipment, it is vital to consider obstacles that might be encoun- tered and proactively address them (see Figure 2). For example, delay at an international border by customs is quite common. For such shipments, consider selecting a courier with experience navigating the regulatory landscape and with services and infrastructure to ensure sample integrity, even during unforeseen delays (e.g., replenishing dry ice, and inclusion of temperature monitoring devices).

\section{Standardizing sample collection procedures across investigational sites}

Building a standardized sample collection procedure that is compatible with all participating clinical sites and ensuring its proper implementation, greatly helps to maintain study sample integrity and facilitates reliable interpretation of biomarker results [6,7]. All participating clinical sites must have trained staff and the critical equipment necessary for sample processing must operate to a standard specification.

\section{Clinical site training}

Patient specimens are an invaluable asset of any clinical development program. Therefore, utmost care must be taken to provide standardized training to clinical site staff prior to the initiation of a clinical trial. A study-specific training manual should be provided, combined with a training session for each participating site by a qualified instructor to ensure the integrity and proper archival of the study samples. Although on-site training is the most effective method of instruction, this may not always be feasible. It is especially true when a large number of

\section{Table 1. Common sources of variability during sample collection and processing.}

\begin{tabular}{|ll}
\hline Issues & Solutions \\
$\begin{array}{l}\text { Lack of trained staff and/or infrastructure for } \\
\text { collection and processing of complex tissue and } \\
\text { blood specimens }\end{array}$ & Careful selection of clinical sites and staff training \\
\hline $\begin{array}{l}\text { Samples are left at } 4^{\circ} \mathrm{C} \text { to clot before centrifugation } \\
\text { resulting in incomplete clotting, and increased risk } \\
\text { of blood cell contamination }\end{array}$ & Provide training and written instruction to sites \\
\hline $\begin{array}{l}\text { For plasma collection: centrifuges in some clinical } \\
\text { sites do not have refrigeration function }\end{array}$ & $\begin{array}{l}\text { The effect of ambient temperature or pre-chilling } \\
\text { the rotor and sample prior to centrifugation should } \\
\text { be assessed and validated }\end{array}$ \\
\hline $\begin{array}{l}\text { Clinical sites do not have -70 }{ }^{\circ} \mathrm{C} \text { freezer } \\
\text { Sample storage must be guided by the stability } \\
\text { results obtained through assay development and } \\
\text { validation. The shipping schedule should reflect } \\
\text { maximum storage time allowable at -20 }\end{array}$ \\
$\begin{array}{l}\text { Excess sample sorting prior to shipping, increasing } \\
\text { potential for thawing of the samples }\end{array}$ & $\begin{array}{l}\text { Samples should be racked or bagged per subject, or } \\
\text { per time point, depending on the analytical needs }\end{array}$ \\
$\begin{array}{l}\text { Customs delays for international shipment of } \\
\text { specimen due to the varied license requirements in } \\
\text { different countries and regions }\end{array}$ & $\begin{array}{l}\text { Select a courier that guarantees replenishment of } \\
\text { dry ice during such delays }\end{array}$ \\
\hline
\end{tabular}




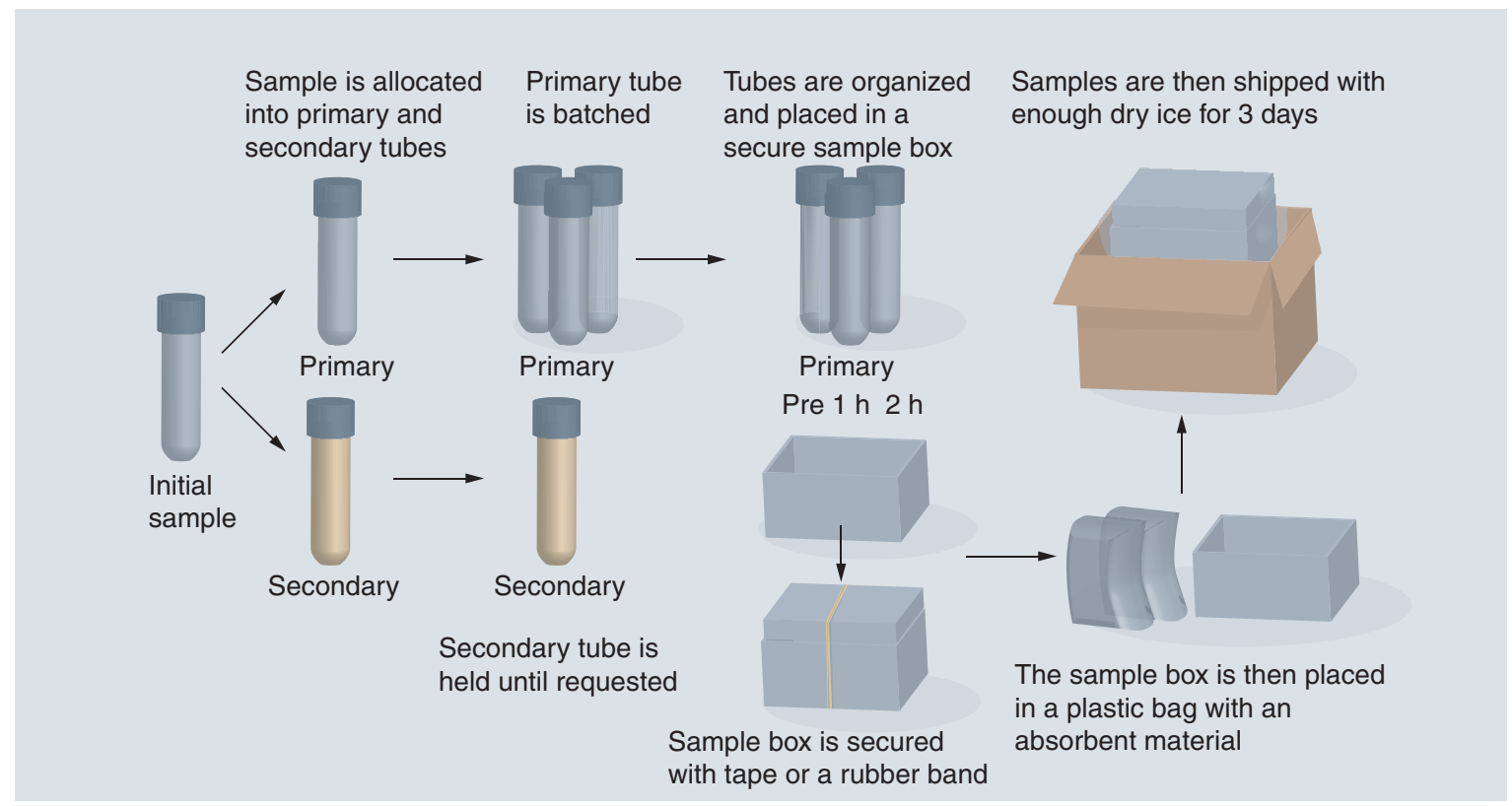

Figure 2. Recommended practice for distribution and shipment of biomarker specimens to mitigate loss and instability.

clinical sites are in diverse geographical locations. In such cases a digital medium such as a training DVD or video meetings may effectively deliver training, as well as provide supplementary or 'refresher' training if required.

Components of a typical training program are described below and summarized in Figure 3, with pertinent guidance documents listed in Table 2.

\section{Essential components of a site training manual} Using the sample collection kit

For uniformity of handling, a sample collection kit containing all necessary components for sample draw and collection should be used by all clinical sites.

\section{Sample processing}

The nature and type of sample matrix (e.g., serum, $\mathrm{K}_{2}$ EDTA-plasma, heparin-plasma) is specified and appropriate collection tubes are provided, together with detailed instructions for their preparation. These include the type of sample collection tube, the processing temperature (e.g., room temperature for serum separation) and centrifugation conditions (e.g., $2000 \times g$ for $10 \mathrm{~min}$ at $4^{\circ} \mathrm{C}$ for plasma separation). For labile biomarkers, special instructions may be provided, for example, blood collection in the presence of protease inhibitors or acidification of plasma. Instructions for documentation of the sample collection time, subject identification, use

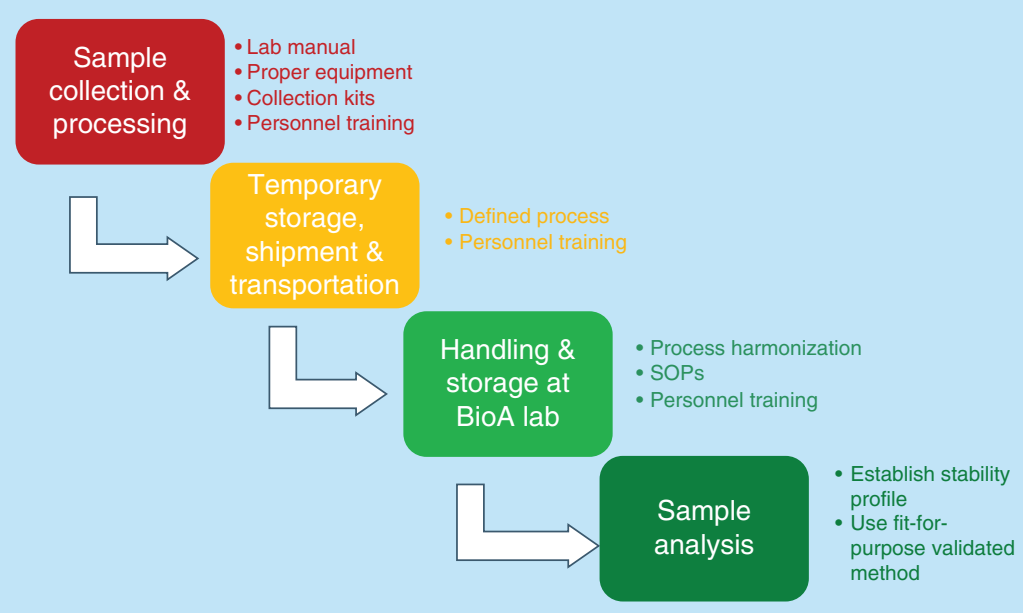

Figure 3. Key elements of a clinical site training program.

SOP: Standard operating procedure. 
Table 2. Pertinent resources and guidance documents.

IATA packing instruction 650 - biological substances, category B

of preprinted labels, placement of samples in a specified order in the storage boxes and preparation of an electronic manifest of the samples should be considered critical parts of the sample collection process.

\section{Temporary storage of samples}

Storage temperature instructions should be provided in the case of temporary storage of samples at the clinical site. It is common practice for samples to be frozen and stored at $-20^{\circ} \mathrm{C}$ at the clinical site then transferred to $-70^{\circ} \mathrm{C}$ storage at the destination laboratory. Hence, appropriate stability evaluation experiments should be designed and executed by the bioanalytical laboratory to cover this practice.

Sample shipment to the bioanalytical laboratory Most clinical samples are classified as biological substances and fall under category B of the schedule of hazardous materials [8]. Therefore, these samples must be securely packaged to conform to the Transportation of Hazardous Materials Regulations of the US Department of Transportation for shipment within the USA or Dangerous Goods Regulations [9] of the International Air Transport Association for shipment worldwide $[10,11]$. Furthermore, the shipper must pay attention to regulations from their local government agencies. Otherwise, shipments may be unnecessarily delayed, possibly causing sample degradation. See Table 2 for pertinent resources.

\section{Specimen packaging}

As a universal rule, study samples for shipment should be packaged following the 'triple packaging system' which consists of: a leak proof primary container housing the study samples; a leak proof secondary container with an adequate amount of absorbent material that can absorb the fluid content of samples in the primary container if leakage occurs - it may also contain dry-ice or cold-pack as needed; a rigid outer packaging that can safely withhold the secondary container with a secure closure, appropriate hazard labels and the addresses of shipper and recipient with necessary documentation.
A copy of the sample manifest can be placed in a waterproof plastic bag and put on the top of the secondary container. Additional guidance for handling hazardous materials is described elsewhere [10].

\section{Key considerations for clinical staff training}

Prior to the initiation of the study, it is essential that a formal training session is held at each clinical site, either in person or via appropriate digital media. Essential components of training may include:

- Review of the sampling regimen as specified in the study protocol. This may include: sampling times, the collection process, any other relevant information in a sample requisition and Good Clinical Practices-specific documentation;

- Detailed discussion of the information provided in the sample collection and handling manual. Procedures for temporary storage of samples at the clinical site should be clearly defined and reviewed with the staff;

- Frequent communications between the clinical site and the receiving laboratory are of utmost importance and the availability of shipment tracking numbers enables both parties to monitor and track the precious shipments. A designated person as a 'single point of contact' also facilitates resolution in cases of discrepancy between the sample manifest and the actual number of specimens received or associated sample demographic information. It is always helpful to provide an electronic copy of the sample manifest to facilitate inventory reconciliation at the receiving laboratory;

- For long-term clinical studies, it may be beneficial to implement scheduled (e.g., quarterly or biannual) training sessions for the clinical staff. These sessions would serve as a refresher for previously trained staff and provide training to new staff members.

In summary, providing sample collection kits together with a sample collection and handling manual, 
followed by formal training of clinical site personnel in advance of the study, greatly minimizes preanalytical variables, in turn preserving sample integrity and maximizing analyte stability. For more complex biomarker collections, biannual training programs may be necessary to refresh existing staff and train new personnel. Refer to [12] for detailed guidance.

\section{Best practices for evaluating stability}

Prestudy stability evaluations should cover the sample handling and storage conditions expected during the conduct of the study, including conditions at the clinical site, during shipment and at all other secondary sites. If clinical sample storage conditions change or samples are held outside of the conditions evaluated during method development or validation, additional stability studies may be warranted to cover these new conditions based on an evaluation of risk and situationspecific considerations [13]. Short-term stability parameters should normally be evaluated within the method validation protocol. Long-term specimen stability studies should only be initiated with a fully developed assay method and in accordance with local laboratory compliance standards. All vendors and lot numbers of assay kits, reagents and analytical standards should be documented and the certificates of analysis retained.

\section{Test plan}

Stability testing should follow an approved protocol that sets out the types of matrix and analytes to be investigated, temperature conditions, periods of exposure, numbers of replicates of each treatment and the analytical method to be used. In the case of tissue samples, it is critical for analytical laboratories to develop a fit-for-purpose storage stability plan as part of the assay validation package.

Recommendations for best practices to assess stability during bioanalyses have been reviewed by Van de Merbel et al. [14].

\section{Preparation of stability samples}

Large quantities of samples are typically required for stability evaluations. When sample is limiting, sample pools rather than individual samples can be used. Stability samples should be prepared using native matrix and endogenous analyte(s) whenever feasible, as recombinant protein may give false results (Figure 4). Appropriate surrogate systems may be considered based on scientific justification. Stability samples should be as close as possible in composition to clinical samples at the time of collection and should be prepared from individuals that are relevant to a study population (e.g., same disease, age). Once collected, samples should either be immediately frozen and stored under the study sample storage conditions (typically $\leq-70^{\circ} \mathrm{C}$ ), or if the analyte is known to be stable at $4^{\circ} \mathrm{C}$, stored refrigerated pending evaluation. It is recommended that stability samples span the calibrated range of the assay or the anticipated clinical range of the analytes. Multiple pools from discrete individuals may be required when native analyte concentration ranges are narrow or interindividual sample stability differences are suspected. If a drug is known to interact with the analyte(s), separate pools should be created to mimic predose/placebo samples and samples from treated individuals.

Aliquots should be prepared using the same volume and storage containers as study specimens. Aliquots are immediately frozen and placed at best storage temperature. After establishment of baseline, samples should be transferred to storage conditions identical to those expected for clinical samples.

\section{Calibration standards \& quality control samples}

Calibration standards (calibrators) are used to quantify the assay and quality control samples (QC) provide an independent check of assay performance. QC sample performance also determines run acceptance or rejection. Calibration standards and QC are prepared by spiking the analyte(s) into a buffer or matrix. Both calibrators and QC are often prepared fresh from stock solutions on the day of testing, but may also be prepared as a ready-to-use series, stored frozen or in a lyophilized form, for example, as part of a kit. Any short-term stability-related aspects of assay performance applicable to fresh standards and QC should have been addressed during assay development and validation. However, demonstrated long-term stability may be required for stock solutions used in calibrator and QC preparation or for ready-to-use reagent standards. Similarly, the stability of calibrators provided in a kit-based assay must be demonstrated by the manufacturer for the shelf-life of the kit if the kit is to be used in stability testing. The use of freshly prepared QC is highly recommended for stability evaluations, especially when pre-prepared frozen standards or kit assay components are used in the assay. This practice avoids errors in quantification of stability samples resulting from erroneous preparations of calibrators or instability of frozen QC. In addition to buffer-based QC, qualified endogenous matrix quality control samples (EQC) are often used during sample testing to monitor the assay's ability to measure endogenous analytes. Use of EQC in stability evaluations may, however, be limited by availability of appropriate stability data to support this practice. In such cases, the assay's ability to measure endogenous analyte must be inferred from test results from the stability samples 
themselves. Caution should be taken in the latter case as it may not be possible to distinguish between sample instability and assay performance issues. Some 'out of control' observations due to poor assay performance may be distinguishable from sample instability when using appropriate control chart tools. For example, a sample falling outside the stability range but returning back into the range in subsequent tests will likely be attributed to assay variability.

\section{Freeze-thaw stability}

During freeze-thaw stability evaluations, the freezing and thawing of stability samples should mimic the intended sample handling conditions to be used during sample analysis [13]. Stability should be assessed for a minimum of three freeze-thaw cycles and frozen at either at -70 and $/$ or $-20^{\circ} \mathrm{C}$ in accordance with clinical study requirements.

\section{Short-term (bench-top) stability}

Bench-top stability experiments should be designed and conducted to cover the laboratory handling conditions that are expected for study samples.

\section{Long-term stability}

The storage time in a long-term stability evaluation should equal or exceed the longest elapsed time from collection to testing of a given sample. A long-term stability study should be initiated as soon as possible after commencement of a validation study. Depending on the timing of the study and if there are known issues with the stability of the analyte (such as many of the smaller peptides like GLP-1 and TGF- $\beta$ ), the scientist may choose to examine sample stability during assay development and repeat sample stability with the validated assay.

\section{Assessment of results}

Sample stability will be demonstrated if the treatment value and the baseline value are within $20 \%$ (or other a priori defined relative error). It is also helpful to monitor the trends of stability evaluations over time and apply control chart methods [15] to identify any out-of control behaviors that could potentially be related to sample stability. Calibrators and QC must meet the method-specific performance criteria. Quality controls must fall within the target concentration range (typically $\pm 20 \%$, or other value defined a priori). Additional requirements may be applied if EQC are included in the stability evaluations (e.g., one out of two sets of EQC at each level must be within the established range of concentrations). Standards, QC and stability samples must pass the method-specific precision criteria (typically, CV $\leq 20 \%$ between replicate wells).

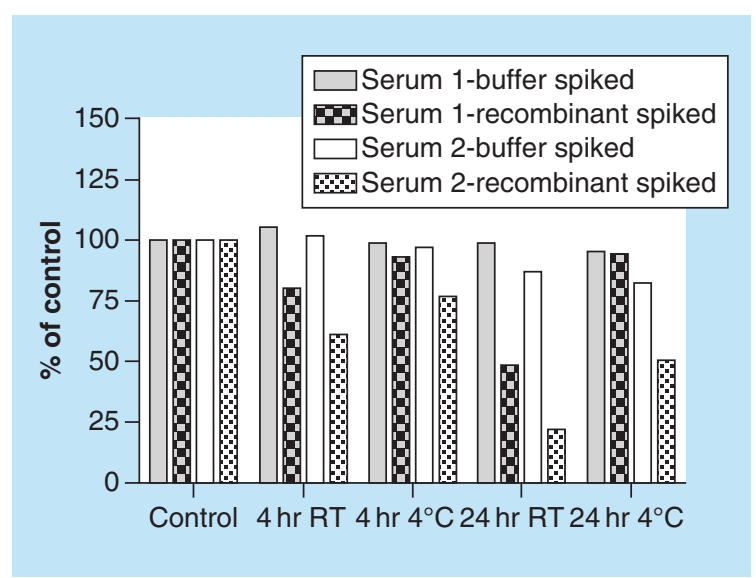

Figure 4. Stability differences between native and recombinant proteins. Two serum samples (serum 1 is an individual sample and serum 2 is a pool of three individual samples) were thawed and spiked with either buffer or recombinant NT-pro-BNP. Samples were aliquoted into polypropylene tubes and frozen at $-80^{\circ} \mathrm{C}$ overnight. One of each of the four samples were removed and left at RT for $24 \mathrm{~h}$, for $4 \mathrm{~h}$ and immediately (control) prior to assay. Samples were assayed for NT-pro-BNP using a Luminex assay. Concentrations were calculated from the standard curve and expressed as the percentage of control sample. RT: Room temperature.

Replicate data points (e.g., wells and tubes) may be excluded from the data if:

- a problem with sample processing or preparation has occurred;

- a system malfunction has been documented;

- at least two results remain available to derive a mean for each pool;

- the result is shown to be an outlier by a statistical test;

- reasons for exclusion of results are documented in the stability study protocol.

Failure of a specific stability parameter does not automatically mean cessation of the study, but rules for immediately repeating a test should be defined in the study protocol. It is good practice to perform the next round of testing at the appointed time after an unresolved stability parameter failure.

\section{Case studies: tissue, blood \& fluid biomarker specimen preservation}

A diverse range of specimens are collected for biomarker analysis. They range from body fluids to tissues, both diseased and normal, and originate from different parts of the body, for example, skin, hair follicles, blood, saliva, urine, cerebrospinal fluid (CSF) 
and so on. Given the significant ethical considerations associated with sample procurement, it is critical to ensure that these valuable specimens are collected and stored with the utmost care to enable generation of reliable biomarker data. This section outlines the authors' experience with collecting, processing and storing some of the common specimen types from clinical trials. To better illustrate the importance of appropriate sample collection, some common pitfalls encountered at clinical sites will be described and ways to mitigate them suggested.

\section{Collection \& storage of tissue biomarker specimens}

Fresh tumor biopsies are much sought-after specimens in cancer trials. The delay time from surgery to specimen preservation could have a significant impact on observation of a 'true' versus a 'false positive' biomarker result. Such delays are a regular occurrence in surgical suites where the primary emphasis is on patient care and adherence to routine hospital practices. Therefore, it is critical to nominate a dedicated individual (e.g., nurse or paramedic) acquainted with the clinical trial to promptly process the specimen following 'easy-to-use' instructions. It is also critical to develop the least-demanding sample-preservation method that will enable discrimination of results (e.g., formalin) or create visual aids for more technically challenging methodologies. A good solution is to post a short video on an easily accessible social media website illustrating the critical elements of the procedure by a laboratory expert. One such example video implemented halfway through a multicenter clinical trial is listed in Table 2. A significant improvement in preservation was observed in the specimens procured postimplementation of this video. Another valuable tool to ensure consistency of sample collection is provision of a simplified checklist covering critical steps (e.g., time to preservation and storage temperature) that can accompany the specimen submitted to the analytical laboratory. Measures to check the quality of specimens received for analysis may also be developed. One such approach involves development of tissue stability indicator protein tests that rely on evaluation of a combination of labile (e.g., phosphoproteins) and stably expressed (e.g., metabolic enzymes) housekeeping proteins [16].

Based on accessibility and their rapid renewal rates, hair follicles and skin punch biopsies are among the more commonly used 'surrogate' tissues to study antiproliferative agents in clinical trials. While the collection of these specimens appears straightforward, more frequently than not, the collection and storage of these specimens has become a hurdle at clinical sites due to lack of familiarity with the final intended use of the specimens. For example, the important end of the follicle for biomarker analysis is the bulb at the base which harbors rapidly dividing cells. Often, specimens with a broken hair shaft are submitted with bulbs missing, primarily due to inadequate training. Other common problems are improper preservation, for example, the bulb not fully immersed in preservatives, incorrect storage temperature, inadequate numbers of hair follicles and varying sites of collection. For instance, arm and scalp hair have vastly different proliferation rates for pre- and post-treatment time points. Many of these issues could be addressed by developing clinical site education materials, for example, visual aids and welldesigned checklists through collaboration between analytical laboratory and clinical operations teams.

Tissue biomarker specimen storage is often neglected during clinical study planning. Tissues typically require extreme temperature storage conditions (e.g., $-70^{\circ} \mathrm{C}$ freezers or liquid nitrogen tanks) to ensure longevity. Most routine hospital environments are devoid of such storage options and, hence, it becomes the responsibility of the sponsor to devise alternative solutions. Two widely used approaches, supported by appropriate analytical laboratory validation are: shortterm storage at $-20^{\circ} \mathrm{C}$ (typically under 30 days) and shipment in special containers to central storage facilities. The latter solution should account for shipment times and dry ice volumes, temperature monitoring devices and other aides, as may be appropriate for the situation.

\section{Collection \& storage of whole blood specimens for biomarker analysis}

Increasingly, blood cells (e.g., peripheral blood mononuclear cells [PBMC], platelets and whole blood specimens) are being utilized as surrogate compartments to measure pharmacodynamic activity of drugs and as receptor occupancy tools for biological agents. However, a problem commonly encountered with blood specimens in clinical trials is the inability to accurately assess biomarker modulation due to changes (e.g., receptor or signaling marker turnover) during the course of specimen shipment from the clinical site to the analytical laboratory. This is a significant problem in the era of modern clinical development that requires inclusion of functional and mechanistic biomarker end points that are best assessed on live target cell populations. Various preservation methods such as Cyto Chex ${ }^{\circledR}$ BCT [17] and proprietary fixative, NovaPerm-3 [18] have been utilized for stabilization of whole cells or individual biomarkers for evaluation by flow cytometry. Some institutions have taken whole blood biomarker utility further by adding a variety of stimulants (e.g., phytohemagglutinin) or phosphatase 
inhibitors to enhance the biomarker signal and mimic the diseased state [19]. PBMC are among the more widely utilized systems to study immune cell subsets and phosphorylated protein biomarkers. Cell preparation tubes provide a versatile one-step method for isolation of PBMC at clinical sites without the need for specialized training. When using either fresh or preserved (fixed) blood or PBMC to assess biomarkers by flow cytometry methods it is important to evaluate the stability of samples (duration, shipping and storage) with cell-type specific markers if possible. Certain cell surface and intracellular antigens are much more labile than others and this factor may influence how long these samples can remain viable. Platelets, on the other hand, are renowned scavengers of angiogenesis markers (e.g., VEGF and angiopoietins) and, hence, widely utilized for monitoring the activity of antiangiogenesis agents [20]. However, isolation of platelets in multicenter clinical trials is a significant logistical challenge, given the need for specialized high-speed centrifuges and a requirement for immediate specimen processing and storage, in turn increasing the cost of the trial.

\section{Collection \& storage of fluid biomarker specimens}

Body fluids are the most common specimens for biomarker evaluations. Each fluid type requires specific procedures and precautions for optimal collection and preservation [7,21].

A common mistake in serum sample collection is to allow blood to clot at $4^{\circ} \mathrm{C}$ before separation. This often results in incomplete clotting, low serum volume and increased risk of blood cell contamination. This error can be avoided by strictly adhering to the guidelines provided by blood collection tube manufacturers. The advantage of plasma over serum is that it can be separated immediately after blood draw, thus minimizing potential changes (e.g., metabolism) that may occur ex vivo and particularly beneficial when analyte stability outside the body is a major concern [22]. Plasma yields are usually higher than serum, possibly due to imperfect clotting. In contrast, serum provides a 'cleaner' matrix, which may improve assay. But the coagulation process consumes all blood clotting factors, which makes serum unsuitable in measuring many biomarkers. Frequent communications between the bioanalyst and the clinical team can aid in the selection of an appropriate matrix for each biomarker assay.

Urine samples are especially prone to circadian cycles. Understanding the intended use of the urine biomarker can help determine the most appropriate time for collection, which in turn should be highlighted in the associated sample collection manual.
Additionally, hydration status greatly influences the concentration of many urine analytes. Measurement of a normalizing analyte, for example, creatinine should be considered in parallel with target biomarker analysis, provided renal excretion rate is considered in the context [20].

CSF collection demands a higher level of skill, which requires trained staff to perform lumbar puncture within a clinical facility. Furthermore, as an aid to interpreting biomarker data from CSF samples, a general CSF analysis should also be performed to include companion tests for proteins, glucose and cells.

Given the wide utilization of body fluids for biomarker studies, it is pertinent to briefly cover agents commonly used to enhance preservation of these specimens. Protease inhibitors and DPP- 4 inhibitors are thought to promote sample stability and are often considered as part of serum and plasma sample collection regimens. However, the practical aspects of having these agents added by individual clinical site technicians must be carefully considered to ensure that immediate and consistent stabilization of blood is indeed achievable in multicenter trials. To help mitigate this risk, checklists or time logs may be implemented to ensure biomarker specimens are collected and processed as intended. Training the clinical staff to accurately pipet fluids also poses an additional challenge. New fluid collection products such as BD P800 tubes, or K2EDTA tubes with added DPP-4 inhibitor can alleviate some processing issues. Furthermore, some sample stabilizers can be added after blood processing to maintain sample stability during storage.

The choice of anticoagulants for plasma collection may impact analyte stability. Heparin plasma is similar to serum in many respects, but without fibrinogen removed. The absence of chelation agents in heparinized blood allows for maintenance of ionic balance, making it most suitable for performance of ex vivo studies. However, heparin tubes are sometimes found to be contaminated with endotoxin [23]. Contaminated heparin tubes can cause cytokine release in the collected blood which can interfere with target analysis. Therefore, careful selection and testing of various lots of tubes prior to the clinical study is desirable. If blood is to be drawn for multiple analyses using different collection tube types, care must be taken to avoid the potential effects of cross-contamination by sharing the same needle for multiple blood tubes containing different anticoagulants and additives. One should refer to the Clinical and Laboratory Standards Institute, which has established the preferred order of blood draw (culture $\rightarrow$ citrate $\rightarrow$ serum $\rightarrow$ heparin $\rightarrow$ EDTA) widely adopted by diagnostic laboratories [24]. 


\section{Conclusion}

Biomarkers can provide objective and quantitative measurement of a biological response to drug treatment that can be modeled to enable rapid dosing decisions in clinical trials in comparison to traditional clinical end points. Given the tremendous interest in the utilization of biomarkers from researchers in academia, industry and nonprofit research organizations, the importance of appropriate preanalytical sample collection and preservation to the integrity of these measurements cannot be overstated.

In this paper, we have dealt with the topics of sample collection, storage and evaluation of sample stability and gathered the authors' collective experience in the field to highlight principal considerations related to these topics and additionally offered general recommendations for best practices. The diverse range of specimen types collected for analysis of biomarkers requires a range of specific tailored strategies to ensure the best possible preservation of the target analytes. Tailoring these preservation strategies involves consideration of a number of crucial and interdependent processes, commencing with patient consent and finishing with specimen testing in designated analytical laboratories. The authors have attempted to capture the salient points for

\section{References}

1 Woodcock J, Woosley R. The FDA critical path initiative and its influence on new drug development. Annu. Rev. Med. 59, 1-12 (2008).

2 Holland NT, Smith MT, Eskenazi B, Bastaki M. Biological sample collection and processing for molecular epidemiological studies. Mutat. Res. 543(3), 217-234 (2003).

3 Betsou F, Barnes R, Burke T et al. Human biospecimen research: experimental protocol and quality control tools. Cancer Epidemiol. Biomarkers Prev. 18(4), 1017-1025 (2009).

4 Vega-Lopez S, Ausman LM, Griffith JL, Lichtenstein $\mathrm{AH}$. Interindividual variability and intra-individual reproducibility of glycemic index values for commercial white bread. Diabetes Care 30 (6), 1412-1417 (2007).

5 Lee JW, Devanarayan V, Barrett YC et al. Fit-for-purpose method development and validation for successful biomarker measurement. Pharm. Res. 23(2), 312-328 (2006).

6 Vaught JB. Blood collection, shipment, processing, and storage. Cancer Epidemiol. Biomarkers Prev. 15(9), 1582-1584 (2006).

7 Tuck MK, Chan DW, Chia D et al. Standard operating procedures for serum and plasma collection: early detection research network consensus statement standard operating procedure integration working group. J. Proteome Res. 8(1), 113-117 (2009).

8 Mayo: Dangerous Goods Training Manual. www.mayomedicallaboratories.com/education/online consideration and stimulate the planning necessary to put sample stability and preservation at the heart of a carefully designed biomarker study. As the general principles outlined in this article are based on actual experiences, it is hoped that the translational medicine community may adopt similar common guidelines as they plan for successful application of biomarker programs supporting future drug development.

\section{Acknowledgements}

The authors thank D Richard Lachno (Eli Lilly and Company, UK), A Mikulskis (Biogen, USA) and R Weiner (Daiichi Sankyo, USA) for valuable feedback and the Ligand Binding Focus Group of the American Association of Pharmaceutical Sciences (USA) for endorsing this activity.

\section{Financial \& competing interests disclosure}

The authors have no relevant affiliations or financial involvement with any organization or entity with a financial interest in or financial conflict with the subject matter or materials discussed in the manuscript. This includes employment, consultancies, honoraria, stock ownership or options, expert testimony, grants or patents received or pending, or royalties.

No writing assistance was utilized in the production of this manuscript.

9 Interstate Shipment of Etiologic Agents www.cdc.gov/vaccines/pubs/surv-manual/appx

10 A Guide To Developing A Hazardous Materials Training Program. www.phmsa.dot.gov/staticfiles/PHMSA/DownloadableFiles

11 IATA Packing Instruction 650 - Biological Substances, Category B. www.iata.org/whatwedo/cargo/dgr/Documents

12 The Center for Clinical \& Translational Science at Ohio State University; education and training resources for clinical research coordinators and staff https://ccts.osu.edu/education-and-training-programs

13 Guidance for industry: Draft Bioanalytical Method Validation. (Revision 1 September 2013) www.fda.gov/downloads/Drugs/Guidances/ucm368107.pdf

14 Van De Merbel N, Savoie N, Yadav M et al. Stability: recommendation for best practices and harmonization from the Global Bioanalysis Consortium Harmonization Team. AAPS J. 16(3), 392-399 (2014).

15 Carroll TA, Pinnick HA, Carroll WE. Probability and the Westgard Rules. Ann. Clin. Lab. Sci. 33(1), 113-114 (2003).

16 Neumeister VM, Parisi F, England AM et al. A tissue quality index: an intrinsic control for measurement of effects of preanalytical variables on FFPE tissue. $L a b$. Invest. 94(4), 467-474 (2014).

17 Davis C, Wu X, Li W, Fan H, Reddy M. Stability of immunophenotypic markers in fixed peripheral blood for 
extended analysis using flow cytometry. J. Immunol. Methods 363(2), 158-165 (2011).

18 Anil Pahuja SS, Caroline Clark, Abdel Saci et al. A novel blood preservation system to study oncogenic signaling pathway biomarkers by flow cytometry in leukemia/lymphoma clinical trials. Presented at: 105 th Annual Meeting of the American Association for Cancer Research. San Diego, CA, USA, 5-9 April 2014.

Ruiz-Medina BE, Ross JA, Kirken RA. Interleukin-2 receptor beta $\mathrm{Thr}-450$ phosphorylation is a positive regulator for receptor complex stability and activation of signaling molecules. J. Biol. Chem. 290 (34), 20972-20983 (2015). Waikar SS, Sabbisetti VS, Bonventre JV. Normalization of urinary biomarkers to creatinine during changes in glomerular filtration rate. Kidney Int. 78(5), 486-494 (2010).
21 Ostroff R, Foreman T, Keeney TR, Stratford S, Walker JJ, Zichi $\mathrm{D}$. The stability of the circulating human proteome to variations in sample collection and handling procedures measured with an aptamer-based proteomics array. J. Proteomics 73(3), 649-666 (2010).

22 Doumas BT, Hause LL, Simuncak DM, Breitenfeld D. Differences between values for plasma and serum in tests performed in the Ektachem 700 XR Analyzer, and evaluation of "plasma separator tubes (PST)". Clin. Chem. 35(1), 151-153 (1989).

23 Newhall KJ, Diemer GS, Leshinsky N et al. Evidence for endotoxin contamination in plastic $\mathrm{Na}+$-heparin blood collection tube lots. Clin. Chem. 56(9), 1483-1491 (2010).

24 Dennis J. Ernst, Lisa O. Ballance, Roger R Calam. Procedures for the collection of diagnostic blood specimens by venipuncture. 27(6), 56 (2007). 